\title{
Experiencias formativas en la etnoeducación de comunidades afrodescendientes en olombia, un proceso en construcción
}

\section{Training experiences in the ethno-education of afro-descendant communities in Colombia, a process under construction}

\author{
DOI: $10.46932 / \operatorname{sjdv} 3 n 1-118$
}

Received in: January $20^{\text {th }}, 2022$

Accepted in: February $1^{\text {st }}, 2022$

\author{
Juliana Sinisterra Quintero \\ Magister en Derecho con énfasis en derecho público \\ Institución: Universidad Del Valle \\ Direccion: Cali, Valle del Cauca, Colômbia \\ E-mail: julianasinisterra@ hotmail.com
}

\section{RESUMEN}

La etnoeducación es la forma de enseñanza y aprendizaje de saberes culturales incluidos dentro de los programas académicos de los colegios y los consejos comunitarios, que permite tener en cuenta la multiculturalidad como parte esencial del modelo educativo tradicional. En Colombia es uno de los procesos culturales y educativos implementados para las comunidades, bien sean indígenas o afrocolombianas. A través del reconocimiento legal de la Ley 70 de 1993 y el Decreto reglamentario 804 de 1995 se logró consolidar un modelo educativo articulado a la educación pública y potestativo a la educación privada con condiciones diversas culturalmente que permitiera, entre otras cosas, el reconocimiento intercultural necesario. Sin embargo, los impactos no han sido muy claros y es que estos deben verse reflejados en dos vías: La generación de un nuevo conocimiento con base en la forma de enseñar y aprender de las comunidades y los efectos que este proceso tiene en la vida económica y laboral de los grupos, que permita dar cuenta sobre cómo funciona, en qué niveles de educación se puede hablar de políticas etnoeducativas y cómo ha sido el trabajo que se ha venido realizando dentro de las comunidades para ejecutar la política. El presente estudio es una apuesta por mostrar los avances sobre algunos de los aspectos más importantes de la política etnoeducativa que se aplica en Colombia a partir de las experiencias formativas de los principales actores, en este caso, las comunidades afrodescendientes.

Palabras clave: etnoeducación, comunidades afrodescendientes, políticas educativas, experiencias formativas, inclusión educativa.

\begin{abstract}
Ethnoeducation is the form of teaching and learning of cultural knowledge included in the academic programs of schools and community councils, which allows taking into account multiculturalism as an essential part of the traditional educational model. In Colombia, it is one of the cultural and educational processes implemented for communities, whether indigenous or Afro-Colombian. Through the legal recognition of Law 70 of 1993 and the regulatory Decree 804 of 1995, it was possible to consolidate an educational model articulated to public education and optional to private education with culturally diverse conditions that would allow, among other things, the necessary intercultural recognition. However, the impacts have not been very clear and should be reflected in two ways: The generation of new knowledge based on the way of teaching and learning of the communities and the effects that this process has on the economic and working life of the groups, which allows to account for how it works, at what levels of education it is possible to speak of ethno-educational policies and how the work has been carried out within the communities to implement the policy. This study is an attempt to show the progress made on
\end{abstract}


some of the most important aspects of the ethno-educational policy applied in Colombia based on the formative experiences of the main actors, in this case, the Afro-descendant communities.

Keywords: ethnoeducation, afro-descendant communities, educational policies, training experiences, educational inclusion.

\section{INTRODUCCIÓN}

Una vez logrado el proceso de reconocimiento político de la etnoeducación, el siguiente paso fue la institucionalización del programa nacional etnoeducativo. En principio, dicho programa contenía un conjunto de componentes: el diseño curricular, la capacitación, la investigación, el diseño, la elaboración del material con el que se iba a enseñar, la asesoría por parte del Ministerio de Educación Nacional, el seguimiento y la evaluación. Propuesta que fue recogida en el decreto 804 de 1994. Sin embargo, dichos planteamientos burocráticos e institucionales, según Rojas \& Castillo (2007), pretendían seguir influyendo de manera coercitiva en la construcción de un nuevo modelo de educación que incluyera la educación propia. Términos semánticos como "seguimiento y evaluación”, "diseño curricular", "materiales educativos" no correspondían propiamente a las prácticas educadoras tradicionales, en cambio, correspondían a las tecnologías propias del control de la educación oficial. Es así como al reflexionar acerca del significado y el impacto de la fusión de la educación propia ancestral y la educación formal, los autores se preguntan ¿es acaso este el modelo que se quería lograr? (Rojas \& Castillo, 2005, p.82).

El modelo etnoeducativo afrocolombiano se constituye como un “heredero" (cursivas añadidas) de los discursos y luchas indígenas, a pesar de que el proceso etnoeducativo de las comunidades afrocolombianas tiene un nacimiento con la ley 70 de 1993, el referente propio con el que se fundó la política educativa constituye una serie de elementos propios de los indígenas. Estas connotaciones conllevan complejidades de fondo y es que la constitución de las comunidades indígenas y afrocolombianas se da a partir de procesos históricos y formas de vida distintas. Las comunidades afrocolombianas son constituidas a partir de procesos heterogéneos. Según Escobar, Grueso \& Rosero (1997), citado en Rojas \& Castillo (2005), hablar de procesos comunitarios afrocolombianos es preguntarse por los patrones de asentamiento rural y urbano, y por las diversas formas de organización y tradición política. Situaciones que complejizan la mirada a la hora de identificar la evolución política educativa para las comunidades afrodescendientes. (Rojas \& Castillo, 2005, p.86).

De ahí que, surta la necesidad de identificar las experiencias educativas que se han podido desarrollar en las instituciones educativas o procesos comunitarios. Por último, se encuentra la identificación conceptual y sus dimensiones, esto permite dar cuenta de los avances en torno a los 
discursos que introduce la política pública y de otro lado los aportes o concepciones comunitarias de los actores sociales.

La metodología propuesta correspondió al análisis cualitativo, mediante la revisión documental de los archivos de prensa, políticas, leyes y artículos de investigación que dan cuenta sobre la creación y evolución de políticas con perspectiva cultural en la educación. Los enfoques teóricos desde los cuales se abordó el análisis corresponden a la discusión entre multiculturalismo e interculturalidad. Por último, es importante mencionar que la presente reflexión hace parte de mi proyecto de investigación doctoral que se encuentra en proceso de construcción, de ahí que, las aproximaciones sean preliminares al problema de investigación aquí desarrollado.

\section{LOS DEBATES, TENSIONES Y PERSPECTIVAS ENTORNO A LA ETNOEDUCACIÓN AFROCOLOMBIANA}

Las tensiones, debates y perspectivas que se encuentran alrededor de la etnoeducación entretejen un concepto cada vez más complejo. En las prácticas sociales de los procesos pedagógicos emprendidos por las comunidades, la concepción sobre la educación es una propuesta por la emancipación, la lucha contra el racismo y la divulgación de sus prácticas ancestrales dentro de los procesos educativos. Aprender de los mayores, cuidar del territorio y conservar la memoria de sus antepasados hacen parte del discurso comunitario construido a partir de procesos empíricos que no precisamente han iniciado por etnoeducadores formalmente constituidos, sino por los propios grupos y comunidades como una necesidad social, política y cultural. En el mismo sentido, los actores sociales, en específico, los maestros, conciben la etnoeducación desde una mirada de "la educación propia que fortalezca la identidad-cultural y al mismo tiempo la promoción de la interculturalidad orientada al reconocimiento, conocimiento y valoración de las diferencias culturales y étnicas de la nación y su inclusión en todo el sistema educativo". (Robinson, 2007, p.17). De otro lado, se encuentran las definiciones legales que se introducen a partir del discurso de las políticas públicas que positivizan o materializan las concepciones de los actores políticos que participan en la construcción de las mismas, aunque no precisamente representan las concepciones que tienen las comunidades sobre estos procesos, pero terminan siendo el discurso oficializado y permitido para concebir y entender dichas prácticas.

La etnoeducación es entendida ${ }^{1}$ como un proceso de recuperación histórica y re significativa para las comunidades afrodescendientes e indígenas. Durante los siglos I al XIX, los procesos de imposición cultural eurocéntrica estuvieron marcados por una educación hegemónica y triangular. Para Meneses (2016), la etnoeducación se inscribe dentro de un marco de luchas y tensiones por eliminar las prácticas

\footnotetext{
${ }^{1}$ Entre múltiples concepciones.
} 
racistas. La concepción comunitaria de los grupos indígenas y las comunidades afrodescendientes está conformada por "las miradas, significaciones, deidades, rituales, historias, espiritualidades, lenguajes, conocimientos y relaciones con el universo de las comunidades”. (p.42). Dicha concepción contribuyó a que dentro de la pregunta por ¿qué es la etnoeducación? Se introdujeran dos perspectivas: el concepto como una mirada endógena y exógena de la etnoeducación. Para Caicedo (SF), citado en Meneses (2016), el concepto bidimensional de la etnoeducación constituye en primera medida, un proceso de entenderla como una educación emancipadora, autónoma, de corte liberal y que lucha por el fortalecimiento del sujeto afro y sus procesos sociales, políticos, económicos y culturales desde la visión de las comunidades afrodescendientes e indígenas.

En segunda medida, es un concepto que va a suponerla como un agente de socialización de la africanidad y la afrocolombianidad con todos los demás sectores de la población colombiana con miras a transformar las de relacionamiento y disminuir el desconocimiento de la contribución de las comunidades en la construcción del Estado-Nación. (Meneses, 2016, pp.43-55). Esta conceptualización implica transformaciones radicales en los procesos didácticos y pedagógicos de los procesos educativos. A pesar de que el MEN ha buscado contribuir a la creación de materiales, enseñanza y aprendizaje de maestros y estudiantes, las transformaciones sustantivas que se basan en la Constitución Política de Colombia (1991) y los procesos que deben encontrarse presentes en la educación cultural: La interculturalidad, el multiculturalismo y la plurietnicidad no han sido del todo implementados. Según Saldarriaga (2006), "la falta de preparación de la escuela para mediar entre la ciencia y la cultura, se pregunta por el rol de los maestros en el rol de formar desde, con y para la diferencia y preguntarse hasta qué punto los formadores están transformados y formados para ejercer una práctica pedagógica desracializada”. (Saldarriaga, 2006, p.57).

Para Rojas \& Castillo (2005) la educación de "los otros"2 corresponde a tres momentos históricos: El primero, es el de la colonización donde se evangeliza la educación general y se invisibiliza las prácticas propias y ancestrales de las culturas indígenas y afrodescendientes. El segundo momento corresponde a lo que los autores han denominado "integracionismo escolar" que permite la institucionalización de las misiones como dispositivo educador en las regiones habitadas por indígenas y afrodescendientes. El último momento corresponde a la positivización de los derechos étnicos, sociales y culturales en las instituciones jurídicas y la inserción dentro de las agendas legislativas. (Rojas \& Castillo, 2005, p.59).

Para Medina (2011) los desarrollos y dinámicas propias de los pueblos indígenas en torno a la educación, antes de la época en que se reconocieran en Latinoamérica y Colombia, fueron iniciativas comunitarias que dieron lugar al nacimiento de lo que hoy se conoce como etnoeducación. Para la autora,

\footnotetext{
${ }^{2}$ Entiéndase indígenas y afrodescendientes y en general, culturas distintas a las eurocéntricas.
} 
dentro de la educación coexisten al mismo tiempo diversos dispositivos de tecnologías educativas que potencializan o reducen los conceptos, prácticas e imaginarios colectivos. En este caso, el instrumento es la escuela. (p.179). Sin embargo, "estos dispositivos gubernamentales no entran a cuestionar las singularidades de la emergencia de sujetos racializados o etnizados, sino que parten de universales antropológicos para atribuir a la ideología y la ausencia o presencia de estructuras mentales e institucionales". (Medina, 2011, p.178). De ahí la imposibilidad por establecer relaciones críticas para construir el ejercicio intercultural dentro de la política etnoeducativa y no solamente la voluntad de los sujetos que conforman el proceso.

Para Enciso (2004), también es necesario entender el proceso etnoeducativo a partir del discurso de la positivización jurídica como política pública del Estado colombiano que inició a partir de 1985, fecha en la que el Ministerio de Educación Nacional (MEN) conceptualizó la política etnoeducativa como un proceso para la capacitación de maestros indígenas y no indígenas ${ }^{3}$, diseño y producción de materiales educativos bilingües, apoyo a investigaciones en lingüística, antropología y pedagogía y la asesoría al seguimiento y evaluación de proyectos regionales. (p.10). Los procesos etnoeducativos como discursos de políticas estatales se han dado a partir de convenios, formación y construcción de currículos académicos dentro de las escuelas de educación preescolar, básica y media. Sin embargo, dichos procesos han pasado por la tensión entre lo que el MEN plantea como viable y lo que las comunidades conceptúan como etnoeducación. "los cambios han traído ventajas y desventajas, progreso y estancamiento, conocimiento e ignorancia, fortalecimiento y debilidad, alegrías y tristezas, contratos y convenios, expectativas no imaginadas". (Enciso, 2004, p.17). Para Pabón, Rojas \& Angola (2011) “mientras las leyes remplacen a la política, es decir, que las demandas educativas sean planteadas en términos de su adecuación o coherencia con el marco legislativo, se seguirá corriendo el riesgo de dejar de lado la correspondencia entre la norma y el proceso histórico que dio lugar a ella”. (Pabón, Rojas \& Angola, 2011, p.103).

Ahora bien, la etnoeducación también es un proceso que debe entenderse a partir del sujeto. Para Popkewitz \& Brennan (2000), “centrarse en el sujeto sin cuestionamientos afecta múltiples temas de poder que se hallan ocultos en las construcciones retóricas”. (Popkewitz \& Brennan, 2000, p.40). Según Meneses (2016), los actores sociales, en específico, “los maestros son sujetos dispositivos del poder hegemónico que no están preparados para transformar las representaciones, imaginarios, concepciones, prácticas pedagógicas y prácticas sociales encaminadas hacia la diferencia”. (p.49). Para el autor, en la medida en que las representaciones sobre afro descendencia de los profesionales de la educación son auto-reflexivos

\footnotetext{
${ }^{3}$ Los procesos indígenas son los primeros en registrarse como procesos etnoeducativos. Sus prácticas pedagógicas como la enseñanza de su lengua o el saber de sus tradiciones hace que la política etnoeducativa inicie en las comunidades indígenas.
} 
en las prácticas pedagógicas, las concepciones, creencias y discursos se podrá lograr una transformación que posibilite la incorporación de nuevas prácticas etno-pedagógicas que humanicen y transformen el contexto escolar para las comunidades afrodescendiente y la diversidad étnico-cultural. (Meneses, 2016, p.63).

\section{LOS DISCURSOS DE LA ETNOEDUCACIÓN CONSTRUIDOS A PARTIR DE LAS EXPERIENCIAS FORMATIVAS DE LOS ACTORES SOCIALES}

El Centro de Estudios e Investigaciones Docentes-CEID-ADIDA ${ }^{4}$ está ubicado en el departamento de Antioquia. Actualmente se encuentra conformado por docentes de varias instituciones públicas y privadas del sector educativo. Tiene como finalidad contribuir al fortalecimiento de la calidad de vida de la educación y de la realidad multicultural del departamento, analizando los problemas étnicos existentes en la educación colombiana y generando propuestas que lleven al reconocimiento, respeto y valoración de las etnias y sus manifestaciones culturales. Según Palacios (2002), constituye además, "un proceso de formación que se pregunta por la identidad y cultura escolar, el estudio cuenta con una metodología mixta $^{5}$ ". En el aspecto cualitativo, utiliza métodos de observación de los intercambios simbólicos cotidianos de los sujetos involucrados y los respectivos criterios etnográficos. En el aspecto cuantitativo, utiliza el procesamiento de datos a través de técnicas estadísticas, para generar así datos que permitan comparar y hacer análisis del proceso etnoeducativo en el plantel educativo. Lo que ha permitido la generación de una propuesta integral estructurada en ejes temáticos: La conceptualización de la etnoeducación como teoría, la eficacia de la legislación etnoeducativa, la aplicación de la cátedra de estudios afrocolombianos y su contexto en los diferentes niveles de educación básica y secundaria, el desarrollo de la diáspora africana y por último, la cuantificación de la población afrocolombiana en los colegios del departamento. (Palacios, 2002, p.78-83).

En igual sentido, el centro etnoeducativo Antonio Maceo se encuentra ubicado en Santiago de Cali en el extremo suroriental de la ciudad y es estudiado por Rentería (2002), quien ha podido evidenciar que sus directivos se han propuesto que los docentes se capaciten en temáticas etnoeducativas, afrocolombianas y pedagógicas, dicha investigación ha permitido evidenciar que, "las propuestas etnoeducativas afrocolombianas permiten ser una alternativa educativa que satisface en gran parte las necesidades socioculturales de las comunas a las que impacta”.(p.82). Además de ser una propuesta en el campo pedagógico que propicia la interacción de prácticas culturales. (Rentería, 20022, pp.81-82).

\footnotetext{
${ }^{4}$ Asociación de Instituciones de Antioquia.

${ }^{5}$ Cualitativa y cuantitativa.
} 
Los estudios realizados por Agualimpia (2002), en la Escuela Normal Superior Demetrio Salazar Castillo de Tadó, ubicada en el departamento del Chocó. Explica de qué forma la población a la que impacta el centro educativo es afrodescendiente en su mayoría e integrada en menor porcentaje por comunidades indígenas, en específico: Emberas, Chamíes y Katíos. Esta institución ha fundamentado su propuesta etnoeducativa en el marco conceptual de la etnoeducación planteada por el Ministerio de Educación Nacional (1993), “como un proceso social permanente de reflexión y construcción colectiva mediante el cual los grupos étnicos fortalecen su autonomía en el marco de la interculturalidad”. (p.84). Para la institución los objetivos generales de la etnoeducación deben consistir en reconocer y difundir los procesos de resignificación, redignificación étnica y cultural de los descendientes africanos en Colombia. Permitiendo reconocer su realidad cultural y aportando a la construcción de la identidad nacional, lo cual aporta de manera significativa a los criterios pedagógicos y didácticos que orientan la educación étnica. A través de procesos investigativos se ha llevado a cabo el encuentro de experiencias tradicionales; recuperación de la historia, práctica de las tradiciones religiosas, difusión de técnicas de explotación minera ancestral, construcción de instrumentos y materiales didácticos, entre otras. Esta investigación ha permitido orientar los procesos de interacción entre comunidades afrocolombianas e indígenas en un mismo espacio. (Agualimpia, 2002, pp.84-85).

La investigación de William Manuel Quiñonez (2002) en el municipio de Barbacoas de la costa Pacífica nariñense en la Escuela Normal Superior la Inmaculada de Barbacoas ha permitido evidenciar los logros que la etnoeducación puede llegar alcanzar, entre ellos: "La formación de bachilleres docentes con énfasis en etnoeducación, la sensibilización de la comunidad a través de la emisora comunitaria “ecos de Telembi", la construcción de un Plan Educativo Institucional intercultural teniendo en cuenta los lineamientos de la Cátedra de Estudios Afrocolombianos”. (Quiñonez, 2002, p.87). En el mismo sentido, se produce la investigación dada por Reyes (2002) en la Escuela Superior Manuel Cañizales de QuibdóA, donde se encuentra satisfactoria la conformación de semilleros de investigación que permiten el desarrollo de pequeñas investigaciones etnoeducativas, entre ellas: El estudio del vocablo afrodescendiente, las prácticas medicinales que ejercen las comunidades, la formación docente, entre otros. Ello ha conllevado la estructuración de procesos investigativos y evidenciado el desarrollo en la formación docente de miembros que hacen parte de la comunidad. (Reyes, 2002, pp. 88-90).

Los procesos de construcción pedagógica y curricular se han trabajado desde la Escuela Normal Superior María Inmaculada, investigados por Mantilla (2002), donde han sido reconocidos a través de principios antropológicos, sociológicos y organizacionales. Según la investigación, "los procesos antropológicos giran alrededor de la persona como proyecto que se construye en relación con los demás. Sociológicos a través de la búsqueda de una nueva sociedad en continuo cambio, donde la cultura se 
extiende como un tejido entre diálogos y nuevos saberes. Aportes pedagógicos, desde el cambio de perspectiva tradicional en la educación por un modelo de educación que atienda a la cultura afrocolombiana”. (Mantilla, 2002, p.91). El diseño curricular ha sido desarrollado a través del Programa Interdisciplinario de Investigaciones en Educación ${ }^{6}$. Por su parte, Cebrián (2016) en su tesis doctoral titulada: Etnoeducación y artivismo: Una tesis doctoral con el colectivo afrodescendiente, plantea que la educación formal para descendientes africanos en la sociedad española ha sido discriminatoria a lo largo de la historia. Las experiencias educativas de la autora le permitieron embarcarse en un proyecto investigativo que busca dar cuenta de las realidades escolares que viven estas comunidades. ¿Cómo se ha podido impactar a través de la historia, la filosofía, la ciencia, el arte y la educación el reconocimiento de sus orígenes y sus formas de vida? Los métodos utilizados para tratar el problema de investigación fueron la acción participante a través de relatos de vida y la observación de la comunidad. Cebrián (2016), "propone ver la etnoeducación con un sentido significativo que promocione la diversidad de valores culturales en las comunidades étnicas, fomente la visibilidad y las identificaciones a través de las obras artísticas de los afrodescendientes y manifieste los intereses y preocupaciones de la comunidad de origen" (Cebrián, 2016).

\section{LA INTRODUCCIÓN AL DEBATE ENTRE EL MULTICULTURALISMO E INTERCULTURALISMO COMO PARTE DEL PROCESO DE LA ETNOEDUCACIÓN AFROCOLOMBIANA}

La etnoeducación comprende la introducción de la cultura como parte de los currículos académicos, los programas que promocionan y motivan las reflexiones culturales y las contribuciones étnicas, los programas de educación antirracista y de reconstrucción y transformación intercultural. Todo esto, permite en conjunto entrever a la etnoeducación como un programa de múltiples aristas entre las que convergen y divergen los actores sociales y académicos que la estudian. Para el MEN, la etnoeducación tiene como principio fundamental o eje rector, la interculturalidad ${ }^{7}$. No obstante, las experiencias educativas y los investigadores que han reflexionado sobre esta categoría divergen sobre esta mirada, de ahí que, la exploración y el debate sobre ¿es la etnoeducación un proceso intercultural o multicultural? Tenga una especial relevancia en el objeto de estudio.

Para Taylor (1993) lo primero que debe pensarse sobre este tipo de cuestiones son las preocupaciones por la identidad y el reconocimiento "la tesis es que nuestra identidad se moldea en parte por el reconocimiento o por la falta de este; el falso reconocimiento o la falta del mismo pueden causar

\footnotetext{
${ }^{6}$ Este diseño se caracteriza por tener una visión holística, integradora y político-social. Donde los alumnos sean capaces de identificar desde una postura crítica la articulación del conocimiento con la realidad.

${ }^{7}$ Decreto 804 de 1995.
} 
daño. Se puede considerar como una forma de opresión que subyugue, deforme y reduzca la identidad". (Taylor, 1993, p.54). Para el autor, "en el plano social, la interpretación de que la identidad se constituye en el diálogo abierto y no por un "guion" social predefinido ha hecho que la política del reconocimiento igualitario ocupe un lugar más importante y de mayor peso". (p.68). De ahí que, el discurso del reconocimiento pueda considerarse en dos niveles; La esfera íntima que comprende la formación de la identidad y del yo como un diálogo sostenido y que siempre se encuentra en debate con los otros. En segunda medida, se encuentra la esfera pública donde la política del reconocimiento igualitario tiene un papel preponderante. (p.69).

Ahora bien, según Kymlicka (1996), el término "multiculturalismo" explora formas muy diferentes de pluralismo cultural, "cada una de las cuales plantea sus propios retos mediante las cuales las minorías se incorporan a las comunidades políticas, desde la conquista y la colonización de sociedades que anteriormente gozaban de autogobierno hasta la inmigración voluntaria de individuos y familias". (Kymlicka, 1996, p.10). Para el autor, lo primero que debe pensarse cuando se construye el término del multiculturalismo es en la coexistencia cultural de diversos grupos que conviven dentro de un mismo estado. La nación es una "comunidad histórica, incompleta pero que ocupa un territorio y comparten una lengua y una cultura diferenciadas. De ahí que, la noción sociológica del término este completamente relacionada con la idea de "pueblo" o "cultura". Para Kymlicka esta noción implica que el estado es multinacional. "Lo que pedimos que se reconozca es la identidad única de este individuo o de este grupo, el hecho de que es distinto de todos los demás. La idea es que, precisamente, esta condición de ser distinto es la que se ha pasado por alto, ha sido objeto de glosas y asimilada por una identidad dominante o mayoritaria”. (Taylor, 1993, p.71).

Para Touraine (1995) citado en Naranjo (SF), el multiculturalismo plantea ciertos conflictividades en los procesos de construcción en las ideas de democracia. En occidente la cuestión de la multiculturalidad solo puede existir en una única sociedad donde el estado estable es el laico y su principio fundamental sea el del pluralismo y la independencia del estado sobre cualquier creencia. Para Touraine, “el multiculturalismo solo tiene sentido si se define como la combinación, en un territorio dada, de una unidad social y de una pluralidad cultural mediante intercambios y comunicaciones entre actores que utilizan diferentes categorías de expresión, análisis e interpretación”. (Naranjo, SF, p.196).

Para Walsh (2005) la multiculturalidad es un término principalmente descriptivo que hace referencia a la multiplicidad de culturas que existen dentro de un mismo espacio o territorio sin que necesariamente tengan una relación entre ellas. (p.5). Atiende a una separación o segregación entre culturas sin un aspecto relacional. Mientras tanto, la interculturalidad, hace referencia "entre culturas", pero no simplemente como un contacto entre ellas, sino un intercambio que se establece en términos 
equitativos y condiciones de igualdad. (Walsh, 2005, p.4). Para la autora, la categoría de interculturalidad comprende la relación de complejidad que entretejen en las interacciones y los intercambios culturales entre personas, conocimiento y prácticas culturalmente diferentes. Preguntarse por la interculturalidad de inmediato se hace alusión a la relación permanente con la identidad. Para Hall (1997), citado en Walsh (2005), la identidad propia y cultural, no es algo que podemos elegir, sino algo que se tiene que negociar socialmente con todos los otros significados e imágenes construidos como conocimientos que nuestro propio (cursivas añadidas) uso de la identidad activan. (Walsh, 2005, p.4).

En contraposición hasta lo que se ha planteado, se encuentra Olivé (1999), para quien el concepto de multiculturalismo hace referencia a "modelos de sociedad que sirven como guía para las decisiones y acciones de los representantes de los Estados, de los miembros de las diversas culturas y en general, de organizaciones no gubernamentales para incluir los aspectos relacionados con los derechos, funciones y obligaciones sobre las relaciones entre los individuos y las diversas culturas. (p.59). Para Raz (SF), citado en Olivé (1999), existen dos tipos de sociedades multiculturales; Las primeras son aquellas que tienen un territorio propio y cuentan con una zona geográficamente definida. La segunda, son aquellas que comparten diversos espacios públicos y servicios con el resto de los individuos o comunidades sin que haya una separación geográfica. (Olivé, 1999, p.57).

Para Silva (2019), el multiculturalismo en América Latina tiene ciertas cuestiones que se plantean como críticas, a pesar que no es un tema que se reduce a la insuficiencia de políticas, su reconocimiento o incumplimiento. Para la autora, "la cuestión es cómo se representa al sujeto, a qué propósitos sirven esas imágenes y cómo estas representaciones son rebasadas por la dinámica sociocultural efectiva, con la cual finalmente colisiona". (Silva, 2019, p.57).

\section{CONCLUSIONES}

La etnoeducación es multiconceptual, depende del actor, en este caso, gubernamental o social, esta será definida de forma distinta. Las principales características conceptuales que corresponden al término han sido -después de realizado el estudio-, las siguientes: La etnoeducación vista desde lo endógeno o denominado casa adentro y la etnoeducación exógena o casa afuera. La primera característica reviste al aprendizaje y enseñanza de la comunidad con la comunidad. La segunda corresponde a las enseñanzas y prácticas que se dan en las instituciones y los consejos comunitarios. Es reiterativo considerar que, si bien son características dentro del concepto hace falta ir más allá. No solamente es hablar de que la etnoeducación también proviene del etno desarrollo ${ }^{8}$, sino que además, debería preocuparse por las categorías que introducen los términos educación, etnicidad, multiculturalidad e interculturalidad, la

\footnotetext{
${ }^{8}$ Bonfil Batalla. (1980).
} 
representación del sujeto y la comunidad, bien sea afrodescendiente o indígena. Así pues, considera la presente investigación que la primera preocupación sobre la mesa es la de ir más allá de lo que usualmente se ha podido catalogar dentro del concepto y la pregunta por la etnoeducación.

Las experiencias educativas que son iniciativas de las comunidades, sus líderes y en general, los actores sociales, son experiencias enriquecidas sobre las prácticas y los saberes ancestrales. No obstante, se reconoce que dichas experiencias carecen del entramado conceptual, curricular y en algunos casos pedagógicos, y se enfocan más en la practicidad de sus saberes. A pesar de que se reconocen algunos procesos titulados "casa adentro" que no son otra cosa que la educación endógena, es decir, la educación propia para sus comunidades, hay ciertas categorías que se dejan por fuera y son de vital importancia para hablar de etnoeducación, esta referencia es importante tenerla en cuenta porque cuando hablamos de etnoeducación afrodescendiente no precisamente estamos hablando de enseñar y aprender el peinado afro o de celebrar solamente el día de la afrocolombianidad, la etnoeducación es un proceso complejo y multiescalar que tiene otras categorías que merecen ser exploradas y hasta la exploración realizada podemos entrever dichos vacíos.

La interculturalidad y el multiculturalismo se encuentran dados en la política pública y los procesos etnoeducativos que inician de las experiencias de los actores sociales que se llevan a cabo Colombia. Esto en primera instancia porque la política etnoeducativa a diferencia de la Catedra de Estudios Afrocolombianos permite la interacción de diversas culturas, las instituciones de educación no precisamente tienen que ser de corte solamente indígena o afro, en ellas, convergen una pluralidad de grupos e individuos diferenciados racialmente. De ahí que, la interculturalidad esté presente. Es multicultural también, porque la política obedece a un campo general educativo que inicia por el reconocimiento multicultural del sistema educativo colombiano y se desarrolla entre la educación ortodoxa y su reconocimiento con la cultura afrodescendiente. 


\section{REFERENCIAS}

Agualimpia, F. (2002). Escuela Normal Superior Demetrio Salazar Castillo de Tadó. Ministerio de Educación Nacional de Colombia. https://www.mineducacion.gov.co/1759/articles85375_archivo_pdf.pdf

Cebrián, A. (2016). Etnoeducación y artivismo: una tesis doctoral con el colectivo afrodescendiente. Pedagogías invisibles. $\quad$ http://www.pedagogiasinvisibles.es/etnoeducacion-y-artivismo-una-tesisdoctoral-con-el-colectivo-afrodescendiente/

Enciso, P. (2004). Estado del arte de la etnoeducación en Colombia con énfasis en política pública. Ministerio de Educación Nacional de Colombia. http://files.afrocolombia.webnode.es/200000031ba2d8ba72f/estado-arte-etnoeducacion-colombia.pdf

Kymlicka, W. (1996). Ciudadanía multicultural. Una teoría liberal de los derechos de las minorías. Barcelona-España. Paidós.

Mantilla, M. (2002). La Escuela Normal Superior María Inmaculada de Manaure. Ministerio de Educación Nacional de Colombia. https://www.mineducacion.gov.co/1621/articles85375_archivo_pdf.pdf

Meneses, Y. (2016). La etnoeducación afrocolombiana: Conceptos, trabas, patriarcado y sexismo, A propósito de los 20 años de la Ley General de Educación 115 de 1994. Universidad Pedagógica y Tecnológica de Pereira. http://www.scielo.org.co/scielo.php?pid=S012272382016000200003\&script=sci_abstract\&tlng=es

Naranjo, G. (SF). ¿Qué es una sociedad multicultural? Universidad de Antioquia. https://dialnet.unirioja.es/servlet/articulo?codigo $=5279750$

Olivé, L. (1999). Multiculturalismo y pluralismo. Ed. Paidós.

Pabón, I. Rojas, A. Angola, J. (2011). Rutas de la interculturalidad. Estudios sobre educación con poblaciones afrodescendientes en Ecuador, Bolivia y Colombia. Enfoques, experiencias y propuestas. Unesco-Quito.

Palacios, L. (2002). El centro de estudios e investigaciones docentes-CEID-ADIDA. Ministerio de Educación Nacional de Colombia. https://www.mineducacion.gov.co/1621/articles85375_archivo_pdf.pdf

Popkewitz, T. Brennan, M (2000). El desafío de Foucault. Discurso, conocimiento y poder en la educación. Ed. Pomares-Corredor.

Reyes, R. (2002). Escuela Normal Superior Manuel Cañizales de Quibdó. Ministerio de Educación Nacional de Colombia. https://www.mineducacion.gov.co/1621/articles-85375_archivo_pdf.pdf

Robinson, D. (2004). La política de la etnoeducación afrocolombiana. Ministerio de Educación Nacional de Colombia. https://www.mineducacion.gov.co/1759/articles-85375_archivo_pdf.pdf

Romero, M. (2010). La educación por y para indígenas afrocolombianos. Ed. Universidad Pedagógica Nacional. https://revistas.javeriana.edu.co/index.php/MAGIS/article/view/3534

Rojas, A. Castillo, E. (2005). Educar a los otros. Estado, políticas educativas y diferencia cultural en Colombia. Ed. Universidad del Cauca. 
Romero, M. (2010). La educación por y para indígenas y afrocolombianos. Ed. Universidad Javeriana. file:///C:/Users/Downloads/3534-Texto\%20del\%20art\%C3\%ADculo-12553-1-10-20120924.pdf

Saldarriaga, V. (2006). Del oficio del maestro de intelectual subordinado a experto subordinador. Revista Educación y ciudad, Instituto para la Investigación educativa y el desarrollo pedagógico.

Silva, C. (2019). Crisis del multiculturalismo en América Latina. Conflictividad social y respuestas críticas desde el pensamiento política indígena. Ed. Universidad de Guadalajara.

Taylor, C. (1993). El multiculturalismo y "la política de reconocimiento". Ed. Fondo de cultura económica.

Walsh, K. (2005). La interculturalidad en la educación. Instituto Internacional de Integración del Convenio Andrés

Bello.

http://repositorio.minedu.gob.pe/bitstream/handle/123456789/3310/La\%20interculturalidad\%20en\%201 $\underline{\mathrm{a} \% 20 \text { educaci\%c3\%b3n.pdf? sequence }=1 \& \text { is Allowed }=\mathrm{y}}$ 